\title{
Mathematical Explanation: A Contextual Approach
}

\section{Sven Delarivière, Joachim Frans \& Bart Van Kerkhove}

Journal of Indian Council of

Philosophical Research

ISSN 0970-7794

J. Indian Counc. Philos. Res.

DOI 10.1007/s40961-016-0086-2

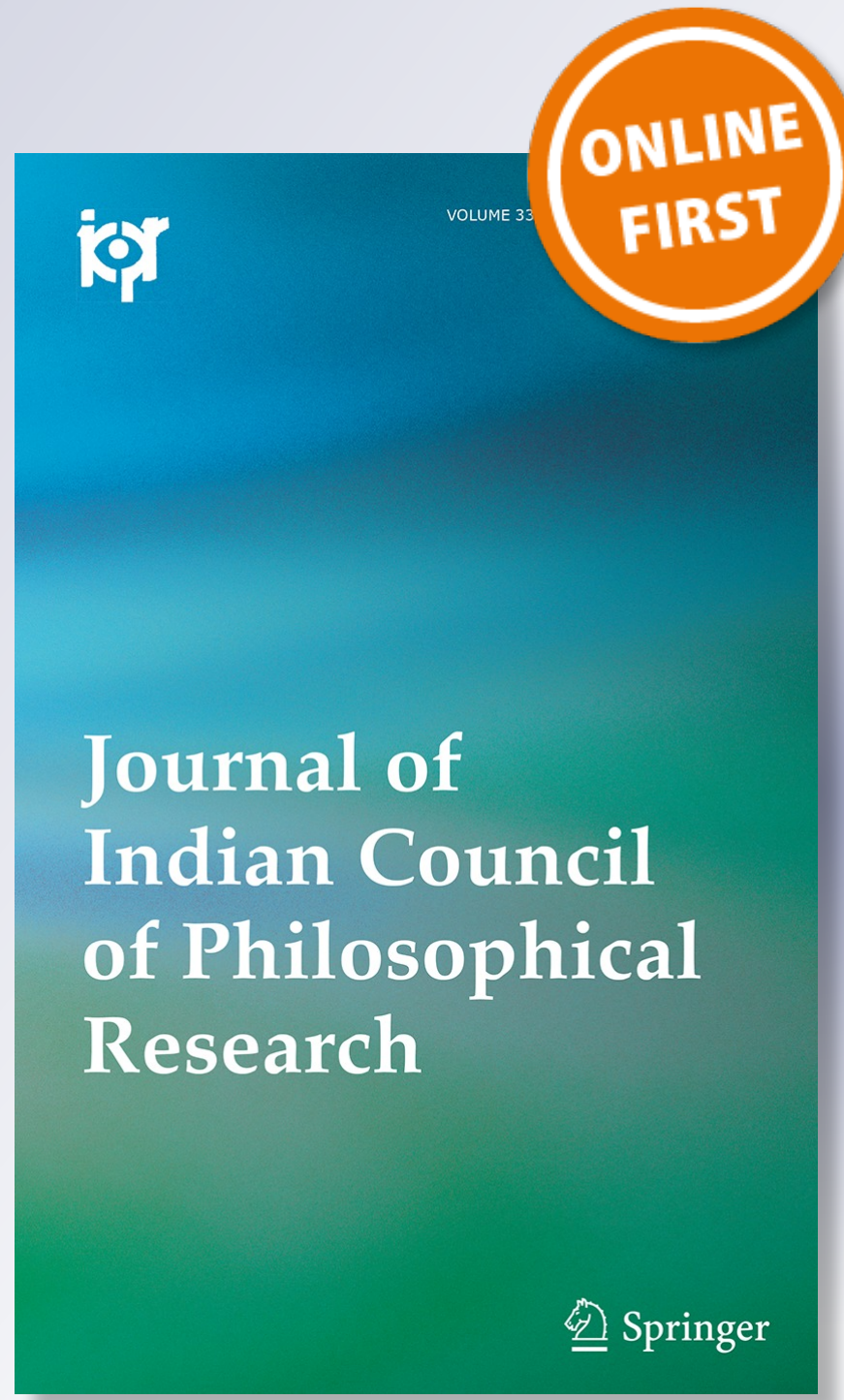

Springer 
Your article is protected by copyright and all rights are held exclusively by ICPR. This eoffprint is for personal use only and shall not be self-archived in electronic repositories. If you wish to self-archive your article, please use the accepted manuscript version for posting on your own website. You may further deposit the accepted manuscript version in any repository, provided it is only made publicly available 12 months after official publication or later and provided acknowledgement is given to the original source of publication and a link is inserted to the published article on Springer's website. The link must be accompanied by the following text: "The final publication is available at link.springer.com". 


\title{
Mathematical Explanation: A Contextual Approach
}

\author{
Sven Delarivière $^{1} \cdot$ Joachim Frans $^{1} \cdot$ Bart Van Kerkhove $^{1}$
}

Received: 18 July 2016/Revised: 26 September 2016/ Accepted: 21 November 2016

(C) ICPR 2017

\begin{abstract}
Purpose In this article, we aim to present and defend a contextual approach to mathematical explanation.

Method To do this, we introduce an epistemic reading of mathematical explanation.

Results The epistemic reading not only clarifies the link between mathematical explanation and mathematical understanding, but also allows us to explicate some contextual factors governing explanation. We then show how several accounts of mathematical explanation can be read in this approach.

Conclusion The contextual approach defended here clears up the notion of explanation and pushes us (at least for now) towards a pluralist vision on mathematical explanation.
\end{abstract}

Keywords Mathematical explanation · Mathematical understanding ·

Contextualism · Pluralism

\section{Introduction}

Mancosu (2008) differentiates between two senses of mathematical explanation, which are both investigated in philosophy of mathematics. The first sense concerns mathematical explanation in the natural or social sciences. Considering the role mathematics plays in our scientific endeavours, clarifying the connection between

Joachim Frans

jfrans@vub.ac.be

Sven Delarivière

sdelariv@vub.ac.be

Bart Van Kerkhove

bvkerkho@vub.ac.be

1 Centre for Logic and Philosophy of Science, Vrije Universiteit Brussel (VUB), Pleinlaan 2, 1050 Brussels, Belgium 
mathematics and the world is an important philosophical challenge. For example, there is an unresolved debate about whether mathematics can genuinely explain a physical phenomenon or merely represent it. Mancosu's second sense concerns mathematical explanation inside mathematics. The underlying idea in this topic is that not all mathematical activity is driven by justificatory aims. For instance, while all proofs show that a theorem is true, some proofs go further and also show why a theorem is true. Several mathematicians and philosophers of mathematics have called the latter an explanatory proof. Philosophical accounts of explanation aim to clarify what it means for a proof to be explanatory. It is this latter sense which we will focus on in this paper.

In "A Contextual Approach", we will outline our contextual account of mathematical explanation. Contextualism comes quite natural, we will argue, once we read explanation as involving a recipient to which it must give understanding (an epistemic, rather than an ontic reading). Because a proof is only explanatory relative to the agent's understanding of it, two contextual factors pertaining to the agent will be discussed: (1) How well a proof transmits understanding, depending on the background/skill corpus $\mathrm{K}$ of the agent, and (2) whether the proof transmits understanding within the particular epistemic interests of the agent.

"Accounts of Mathematical Explanation" explores those same ideas by applying them to several accounts of explanation, namely those of Steiner, Cellucci and Lange. We show an epistemic and contextual reading of each of them and give an illustration from mathematical practice.

The reason we discuss not one but several accounts of explanation will be argued for in "Embracing Pluralism". No single account of mathematical explanation has, at present, managed to capture everything that is mathematically valuable about an explanatory proof. Ergo, we believe it unwise, at present, to limit ourself to any one of them and would rather accept a (heuristic) pluralism about the accounts of mathematical explanation to be considered in dubbing a proof as explanatory. Furthermore, we believe the value of a type of mathematical understanding (and the type of explanation that grants it) may themselves vary with context, which could be expressed as a third contextual factor.

\section{A Contextual Approach}

\section{The Ontic and Epistemic Distinction}

Before we explore accounts of explanation, we believe it fruitful to this phenomenon from the perspective of the ontic/epistemic approaches. This is a distinction borrowed from Salmon (1984) in the debate about scientific explanation. Within the literature on mathematical explanation, talk about epistemic or ontic approaches is absent. Our aim here is to use the epistemic approach in order to clarify some relevant aspects about the study of mathematical explanation that have hitherto remained overlooked or neglected. The ontic conception, following Salmon, sees explanations as 'exhibitions of the ways in which what is to be explained fits into natural patterns or regularities' (1984: p. 293), while 'the epistemic conception takes scientific explanations to be arguments' (ibidem). More generally, ontic versions of explanation see the locus of explanation as that which 
produces the explanandum, while epistemic versions see the locus of explanation as that which makes the explanandum intelligible to an inquirer.

In philosophy of science, ontic views take scientific explanation to be fully objective things in the world, distinguished from the statements or arguments reporting this actual explanation. For causal accounts of explanation, this means a given phenomenon is explained by the set of causal facts, and not by the scientific representation of these facts. ${ }^{1}$ Epistemic views, on the other hand, believe that scientific explanation can only take the form of communicative acts, texts or representations. These views see explanation as a human activity that is deeply connected with understanding. Rather than being full-blooded things in the world, explanations have the purpose to increase our knowledge about the world.

A mathematical proof can be seen as an argument by which one convinces oneself or others that something is true, so it might seem hard to go beyond epistemic talk about an explanatory proof. However, while the content of any particular proof is the fruit of a person's epistemic work, it can be separated as an object independent of a particular mind. Other people can read this proof and be convinced by it. This leads us to the question whether showing why a theorem is true is a feature of the proof itself or a feature of communicative acts, texts or representations. This can be fleshed out as the difference between ontic and epistemic approaches:

An account of explanation is ontic if it states:

Proof $P$ of theorem $t$ has explanatory value if and only if $P$ itself is the explanans of $t$ regardless of whether it gives understanding to any particular agent.

An account of explanation is epistemic if it states:

Proof $P$ of theorem $t$ has explanatory value if and only if the explanans consists of arguments (in the broad sense) including $P$ that grants understanding of $t$ for a particular agent $\mathrm{S}$.

This distinction has consequences for the study of explanatory proofs in mathematics. While not every agent will be capable of grasping the explanation, this fact does not change the explanatoriness of the proof for the ontic account. Ontic accounts have to specify which characteristics a proof has or lacks in order to distinguish explanatory from non-explanatory proofs. The truth of 'Proof $\mathrm{P}$ is explanatory' is independent of a particular recipient's (epistemic agent) response to (i.e. understanding of) it. This leads to the following statement: ${ }^{2}$

An account of explanation is ontic if there can be no statement:

'Proof $P$ explains theorem $t$ ' is true relative to person $\mathrm{S}$ and not true relative to person $\mathrm{S}^{\prime}$.

\footnotetext{
${ }^{1}$ See, for example, Craver (2014: p. 40).

2 As inspired by Khalifa (2016).
} 
This is not the case for epistemic accounts of mathematical explanation. Like the ontic approach, the characteristics a mathematical proof has (or lacks) can and should still be taken into consideration in the epistemic approach, but, unlike the ontic approach, they always have an agent's response (i.e. understanding) coupled to them. Since we only speak of explanation if the agent reaches understanding, the answer to the question whether a proof is explanatory does not proceed in the same way for the epistemic as it does for the ontic approach. The truth of 'Proof $\mathrm{P}$ is explanatory' is relative to the particular recipient's response to (i.e. understanding of) it. This leads to the alternate statement:

An account of explanation is epistemic if there can be a statement:

'Proof $P$ explains theorem $t$ ' is true relative to person $\mathrm{S}$ and not true relative to person $\mathrm{S}^{\prime}$.

It is important to mention that ontic approaches do not necessarily deny the relation between explanation and understanding. In fact, 'virtually every theory of explanation also places a premium on the power of an explanation to produce understanding' (Trout 2005), but the crucial difference is that, for the ontic approach, that relation does not play the role of identifying something as an explanation. It may, for example, be the case that there is an explanation, but nobody understands it. Epistemic views, as we presented them, do start with the idea that there is a fundamental relation and that it is thus meaningless to speak of an explanation in that case. In this paper, we will build upon an epistemic approach to mathematical explanation.

\section{Understanding}

In spite of the intimate link between explanation and understanding, philosophers have had doubts about the philosophical relevance of the notion of understanding. A major cause of concern is the assimilation of understanding with the subjective 'ahafeeling'. This concern has been raised by Hempel (1965) and Trout (2002), who state that the feeling of understanding is not at all a reliable cue for genuine understanding. They argue that this feeling of understanding might be an interesting topic for psychology but has no place in the philosophical analysis of explanation. The feeling of understanding may be its most familiar and salient aspect, but it is almost unanimously agreed that it is neither necessary nor sufficient for genuine understanding (Wilkenfeld 2013).

Waskan (2011) instead argues for a 'success' interpretation of (scientific) understanding which goes beyond merely feeling we understand the world. How we determine understanding as successful, whether it is in science or mathematics, is a philosophical and not a psychological topic. Most positive accounts of understanding either explicitly (Avigad 2008; Sierpinska 1994) or implicitly (de Regt and Dieks 2005) defend a functional 'success' interpretation: When an agent $S$ understands $\mathrm{X}$, we attribute that agent certain abilities concerning $\mathrm{X}$. While a number of people equate understanding with mental states (or representations) instead of abilities, abilities are invariably present as part of the account, be it as 
symptom or trait. We do not wish to deny mental states playing a role in understanding, but, to characterize success, we find more solid ground in the possession of abilities as opposed to inaccessible, theoretical mental states. In short, we can attribute understanding to someone with successful abilities without knowledge of mental states, but not vice versa. This leads to the following definition of understanding that we shall be working with:

'Agent ${ }^{3} \mathrm{~S}$ understands $\mathrm{X}$ ' corresponds to 'agent $\mathrm{S}$ possesses particular abilities related to $\mathrm{X}$ '.

Having defined understanding in such a way entails that we can (in principle) list all abilities relevant to understanding. Avigad (2008) has listed some (general) abilities involved in understanding a mathematical proof, such as: responding to challenges as to the correctness of it, identifying key features, identifying the nature of the objects and questions, mustering the relevant background knowledge, exploring the space of possibilities fruitfully and so on. The particular object of understanding under consideration in this paper is that of a theorem and more specifically understanding why the theorem is true. This corresponds to what an explanatory proof grants. Combining this reading of understanding with the epistemic reading of explanation, we get:

A proof $P$ of theorem $t$ has explanatory value if and only if it grants agent $\mathrm{S}$ abilities appropriate to understanding why $t$ is true.

Since we have said that an explanatory proof $\mathrm{P}$ of theorem $\mathrm{t}$ is one which gives understanding of why-t (relative to an agent), it follows that an explanatory proof is one which grants the agent abilities appropriate to understanding why-t. ${ }^{4}$ Further clarifications will follow once we discuss examples of accounts of explanation.

\section{Contextualism}

A benefit of adopting the epistemic approach is that it gives a perfect set-up to make context-dependent claims about explanation. For the ontic approach, the truth value of 'Proof $\mathrm{P}$ explains theorem $\mathrm{t}$ ' is absolute, meaning proof $\mathrm{P}$ would explain theorem $\mathrm{t}$ (or not) for all agents. Under the epistemic approach, absolutes are (in principle) possible, meaning there can exist a $\mathrm{P}$ which gives understanding of why theorem $t$ is true to all agents, but this need not at all be the case. In fact, it clearly is not the case for a lot of proofs; not every mathematician attains the same abilities from them. Given this, we are in need of a contextual reading of 'Proof $\mathrm{P}$ explains theorem $\mathrm{t}$ '

\footnotetext{
3 We use 'agent' in a broad enough sense to include all entities with non-trivial mathematical intentions and actions, being they a single human mathematician, a cooperative community or a sophisticated automated theorem prover.

4 This leads to the following difference between the two: While understanding is the possession of abilities, explanation is the message that transfers them. This implies that having an explanation leads to understanding (for agent S), but does not imply the converse. An agent who understands will have the abilities granted by an explanation, but does not necessarily have an explanation (at least in the messageready sense, although the substance is certainly present).
} 
that allows us to specify when and why abilities are attained. In that spirit, we now wish to present two contextual factors.

\section{Contextual Factor K (Background/Skill Corpus)}

In order to grasp (or develop) the argument of a proof, an agent needs to have a background/skill corpus fertile for it. The skills and background that the agent possesses are crucial elements in determining whether a proof will be successful in providing understanding. In the context of a particular agent $\mathrm{S}$ and more specifically its background/corpus $\mathrm{K}$, the explanatory value of a proof can be high because the specific features of the proof match his or her specific background and/or skills in such a way that an understanding why the theorem is true can be attained. For another agent $\mathrm{S}^{\prime}$, with another corpus $\mathrm{K}^{\prime}$ (i.e. another context), the explanatory value of the same proof might be weaker, if not entirely absent, simply because the agent $\mathrm{S}^{\prime}$ misses relevant background knowledge and/or skill to work with the proof. Note that we are not hereby saying that an explanatory proof must necessarily be the easiest or the most readily accessible. We do not see background/skill corpus $\mathrm{K}$ as static, thus allowing the possibility for a proof to become explanatory in lieu of some work. However, not until the work is done and $\mathrm{K}$ is fertile, will the proof be properly explanatory. In short, the explanatory value of a proof needs to be evaluated in the light of an agent's background/skill corpus $\mathrm{K}$. This is a sort of 'success' criterion for understanding based on an proof/corpus couple (P, K).

What we've said so far is that proof $\mathrm{P}$ won't be sufficient for granting understanding across all agents. However, we have reason to believe a particular proof P need not be necessary either. Nothing forces us to think the ability/abilities of understanding why a theorem is true can only be attained by a single proof. The same ability may be attained by different proofs (for different or even the same agent). In short, success depends on the agent $S$, by virtue of the couple $(P, K) .^{5}$

\section{Contextual Factor I (Epistemic Interests)}

We have mentioned that explaining why a theorem $t$ is true can be translated to granting abilities appropriate to understanding why-t. If one would need to list all the abilities that are appropriate to understanding why-t, this would involve variations among several domains, ${ }^{6}$ not all of which are of equal interest to every agent. If an agent has interests within a particular domain, then we call those epistemic interests and we denote them with I. I is then a particular perspective of questions to look at t. Having I as a contextual factor can help specify which abilities appropriate to understanding why $t$ is under consideration. Generally, we expect an agent to have epistemic interests which successfully fit with their background/skill corpus, but this needn't be so. An agent may have interests outside

\footnotetext{
${ }^{5}$ We call the contextual factor $\mathrm{K}$ without mention of $\mathrm{P}$ because the sentence under consideration is 'Proof P explains theorem t', which holds $\mathrm{P}$ constant. If the sentence was 'Theorem $\mathrm{t}$ is explained', then the couple $(\mathrm{P}, \mathrm{K})$ would be the contextual factor.

${ }^{6}$ There are different ways by which domains can be differentiated, e.g. by historically, by school of thought, by mathematical subdisciplines.
} 
of their (current) background/skill. Therefore, it seems appropriate to have I as a separate contextual factor.

Two agents $\mathrm{S}$ and $\mathrm{S}^{\prime}$ may have different epistemic interests $\mathrm{I}$ and $\mathrm{I}^{\prime}$, each of which specify different abilities that will be sufficient to understanding why theorem $\mathrm{t}$ is true. Whether $\mathrm{P}$ explains theorem $\mathrm{t}$ for $\mathrm{S}$ will depend not only on the success of the couple $(\mathrm{P}, \mathrm{K})$, but on whether the success captures the epistemic interests $\mathrm{I}^{7} \mathrm{~A}$ couple of illustrations are given in "Accounts of Mathematical Explanation". In short, the domains that are of interest can differ with context I and specify which abilities involved in understanding why-t are important. We propose, therefore, that the explanatory value of a proof to be evaluated in the light of epistemic interest I.

\section{Accounts of Mathematical Explanation}

In this section, we look at the state of the art in the literature on mathematical explanation. One of the most influential accounts, leading to a renewed interest in the topic mathematical explanation during the last few decades, is provided by Steiner (1978). Cellucci (2008) and Lange (2014) propose two alternative accounts. These three accounts will be presented in this section. As we will argue, each of these accounts can be read from the epistemic and contextual approach developed earlier. In order to display the legitimacy of our contextual approach, we will present a case for each account highlighting the role of epistemic interest in explanatory endeavours.

\section{Steiner}

\section{Account}

To draw a distinction between explanatory and non-explanatory proofs, Steiner (1978) introduces the notion of a characterizing property: a property unique to a given entity or structure within a family or domain of such entities or structures. An explanatory proof is a proof that depends on a characterizing property of an entity or structure mentioned in the theorem. But merely pointing to this characterizing property is not enough. One must be able to generate new, related proofs by varying the property (substituting it with the characterizing property of a related entity) while holding the proof idea constant. As an example, we present a proof of the Pythagorean theorem, which Weber and Verhoeven (2002) offer as a case fitting Steiner's account.

Theorem For a right triangle with legs $a$ and $b$ and hypotenuse $c, a^{2}+b^{2}=c^{2}$.

\section{Proof}

(1) For every triangle $A B C: a^{2}+b^{2}-2 a b \cos (a, b)=c^{2}$.

\footnotetext{
7 If epistemic interests can differ, we have an additional reason why different proofs can, contextually, be of explanatory value—namely that they render abilities belonging to different epistemic interests.
} 
(2) For every angle $\theta: \cos (\theta)=0$ if $\theta=90^{\circ}$.

(3) For every right-angled triangle $A B C$ with hypotenuse c: $(a, b)=90^{\circ}$.

(4) Hence, for every right-angled triangle $A B C$ with hypotenuse $c: a^{2}+b^{2}=c^{2}$.

This is both a valid proof of Pythagorean theorem and fits the criteria provided by Steiner to count as an explanatory proof. First of all, it makes reference to the characterizing property of an entity mentioned in the theorem, namely the fact that a right-angled triangle has an angle that equals $90^{\circ}$. This identifies it uniquely in the family of triangles. Moreover, the proof depends on this fact in the sense it is used to deduce the Pythagorean theorem from the law of cosines. Finally, we can generate new related theorems by holding the proof idea constant and varying it with the characterizing properties of other entities of the family. For example, we can derive that for all obtuse triangles $c^{2}>a^{2}+b^{2}$.

\section{Ontic and Epistemic Reading}

Let us take a step back and present both an ontic and an epistemic reading of Steiner's account. We stated that an ontic account classifies a proof as having explanatory value by only looking at features of the proof itself. A proof is explanatory if it fits certain criteria, meaning that the proof itself is the explanans of the theorem. For Steiner, this would entail the following two criteria:

- The proof makes reference to, and depends on, a characterizing property of an entity or structure that is mentioned in the theorem.

- The proof is deformable, meaning that replacement of the characterizing property with a characterizing property of a related entity or structure leads to a related theorem.

An epistemic account classifies a proof as having explanatory value if and only if the explanations are arguments including $\mathrm{P}$ that grant understanding of $\mathrm{t}$ for a particular agent. For Steiner, this would entail the following arguments/activities:

- Identifying an entity or structure mentioned in the theorem and a family of related entities or structures.

- Inspecting the proof and indicating what characterizing property is used in the proof.

- Seeing how the theorem changes in response to replacing the characterizing property with characterizing properties of entities or structures from the family.

What gives the explanation, in this view, is not merely the proof, but a family of related proofs and an argument which highlights a specific aspect of each proof that makes them related in a particular way. We also stipulated that, under the epistemic reading, an explanation gives understanding, where understanding was seen as possessing particular abilities related to the theorem. Our reading of Steiner's 
account can lead to an agent possessing some abilities out of this (not exhaustive list $^{8}$ of):

- The ability to see that the theorem holds for a specific entity or structure within a family of entities or structures.

- The ability to motivate how a specific property of this entity or structure contributes to the truth of the theorem.

- The ability to give a similar argument for theorems about related entities or structures.

The analysis of Steiner's account through these two approaches reveals a tension in it. Steiner does not talk about an agent or the contextual factors that are related to introducing an agent in his account of explanation. Neither are epistemic benefits, such as understanding, discussed. This seems to suggest, without making this explicit, that Steiner has an ontic view in mind. Alternatively the requirement for a characterizing property to be found and a proof to be deformable suggests that actual activities or arguments from an agent are part of the explanans.

\section{Contextual Factors $K$ and $I$}

The agent's skills and background knowledge are crucial elements in our epistemic and contextual framework to mathematical explanation. This can be fleshed out in our adaptation of Steiner's account as well. Without knowledge in geometry and trigonometry, and the skill to manipulate symbolic statements, one would be incapable of constructing or grasping the explanation of the Pythagorean theorem as sketched above.

Moreover, a specific epistemic interest gives voice to this explanation. Instead of searching for the ability to give a similar argument for the Pythagorean theorem and the related theorem(s) about the relation between squares constructed on the sides of acute or obtuse triangles, one could be interested in giving similar argument about areas of the faces of a tetrahedron in three-dimensional Euclidean argument. In that case, another argument would be given, possibly based on another proof. The fact that there are multiple ways to characterize a mathematical entity or structure is something Steiner already recognized:

We have thus a relative notion, since a given entity can be part of a number of differing domains or families. Even in a single domain, entities may be characterized multiply (Steiner 1978: p. 143. Emphasis added).

But while Steiner seemingly concludes that all the proofs that fit his criteria are explanatory, we propose that explanatory value of a proof needs to be evaluated in the light of the agent's epistemic interest. We give an example from mathematical practice in the following section.

\footnotetext{
8 An exhaustive list, if possible, would include the ability to (re)construct a proof of the theorem, but, with the following list, we wish to draw focus on the abilities beyond justification.
} 


\section{Illustration from Practice}

As an example, we look at the different approaches from Dedekind and Kronecker, who both published pathbreaking works on algebraic number theory. Roughly at the same time, they tackled the same problems, but each within their own characteristic approach:

What should not pass unnoticed, however, is the very existence of two theories that developed at the same time as answers to one and the same question, but which nevertheless display very different perspectives and spirit. Kronecker and Dedekind were faced with the same body of mathematical knowledge, but approached it through different systems of well-defined and consistent images of knowledge; their contributions to the consequent expansion of the body of knowledge were sensibly different (Corry 2012: p. 120).

It is hard to compare the incommensurable background assumptions of these two mathematicians. These differences can be located at the metaphysical level, for example Dedekind's choice to use axiomatic notions and the infinite as opposed to Kronecker's view on mathematics as constructive and finitary. Reck's (2009) analysis of Dedekind's work, as well as its relation to understanding, includes a comparison between Dedekind and Kronecker on the local level of proofs. Reck uses Steiner's account of explanation for this investigation. Moreover, Reck also sees successful explanation as what improves our understanding, which ties in nicely with our reading of Steiner. What is most interesting for the scope of this paper, however, is how Reck illustrates that starting from the same question 'Why are certain kinds of algebraic equations solvable by integers while others aren't?', Dedekind and Kronecker differ in the way they would give their explanation:

For Kronecker, the contrast class consists of a tightly circumscribed range of equations, corresponding to number fields constructed finitistically; and the presupposed factors are computational ones. For Dedekind, the contrast class is determined by an enlarged class of number fields, thus consisting of a larger number of equations; and the relevant explanatory factors involve entities defined set theoretically and considered structurally. Altogether, the most radical differences between Dedekind's and Kronecker's approaches can be located at this level, I would suggest. They consist of differences in the general background assumptions for their respective explanatory enterprises (Reck 2009: p. 165).

Some further clarification is needed. Reck uses the notion of contrast class, a notion often used in discussions about scientific explanation. ${ }^{9}$ The goal of specifying a contrast class is showing why something, rather than an alternative, is true. This is analogous to Steiner's idea of a related family of structures and entities. The contrast class is what differs for Dedekind and Kronecker. The quote also mentions relevant explanatory factors, which likewise point to different preferences of where the explanation needs to be searched. Evidently, this has an

\footnotetext{
${ }^{9}$ See, for example, van Fraassen's (1980) pragmatic theory of explanation.
} 
effect on which characterizing properties are considered. We argue that these differences are due to distinct epistemic interests.

Steiner's account revolves around the questions of what is considered as a family of related entities or structures and what is considered as a characterizing property. In our view, this can be filled in different ways depending on the context. Rather than stating that all proofs that fit certain criteria are explanatory proofs in itself, some proofs will be explanatory for Dedekind, and other proofs will be explanatory for Kronecker. Further investigation is needed to get a better grip on specific cases, and these can be used to adjust Steiner's account where necessary. But the case of Dedekind and Kronecker nicely illustrates how descriptive claims about the explanatory value of a proof should always be accompanied with specification of an agent's skill and background knowledge and his/her epistemic aims.

\section{Cellucci}

\section{Account}

Cellucci (2008) argues that explanatory reasoning, which aims to show why something is true, is connected with the analytic method. The core of the analytic method is that one solves a problem by making (plausible) hypotheses, usually obtained by a mixture of both deductive and inductive inferences. The idea is to find a hypothesis that is a sufficient condition for the mathematical problem. Hypotheses are always subject to revision. Cellucci contrasts this kind of reasoning with the axiomatic method, which consists of obtaining a result from axioms and rules of inference. In this method, axioms are to be taken as absolutely true. Cellucci claims that this kind of reasoning only shows that something is the case without showing why. In terms of the analytic method, Cellucci also argues what an explanation would then consist of:

A hypothesis provides an explanation of a problem if it plays an essential role in solving it, in the sense that it reveals an aspect of the problem that is essential to a solution of the problem. In that sense, an explanatory hypothesis is strictly connected with the problem (Cellucci 2008: p. 206. Emphasis added).

An example Cellucci discusses is the problem to demonstrate the Pythagorean theorem (Cellucci 2008: p. 207). To solve this problem, he proposes, within a wider argument, to use the hypothesis that the sum of three interior angles of a triangle is equal to two right angles. What is crucial for Cellucci is that this hypothesis is not only capable of solving the problem at hand, but plays an essential role in solving the problem. In this way, it explains why the Pythagorean theorem is the case. This is not the case for all arguments that lead to the Pythagorean theorem: 'Thus, although the Pythagorean theorem can be proved from the axioms of set theory, the latter do not provide an explanation of the problem posed by the Pythagorean theorem since they play no essential role in solving the problem [in the sense it reveals an aspect of the problem essential to its solution]' (Cellucci 2008: p. 206). 


\section{Epistemic Reading}

Cellucci's account can be interpreted in our epistemic approach as well. This would go as follows: Firstly, what gives the actual explanation goes beyond proof. A broader argument is given by:

- Identifying the exact role of premises and/or auxiliary assumptions (instead of treating them as a given).

- Identifying how a premise or an assumption reveals an aspect that is essential for producing the theorem.

This can be restated as abilities concerning understanding why the theorem is the case. Abilities that are central to this account are:

- The ability to motivate which premise or auxiliary assumption is essentially connected with the theorem.

- The ability to highlight an essential aspect this premise or assumption reveals that leads to the theorem.

\section{Contextual Factors $K$ and I}

Cellucci can also be read in our contextual framework. In order to highlight the relevance of a step, an agent has to have certain knowledge and skills (contextual factor $\mathrm{K})$. Moreover, the agent's epistemic interest (contextual factor I) can have an influence on what is seen as essential or not. Cellucci seems to acknowledge this possibility:

That the very same thing may have distinct reasons, thus distinct explanations, depending on the fact that problems have many sides, so they can be seen from distinct perspectives, each of which suggests a distinct hypothesis, thus a distinct explanation. Any hypothesis establishes a connection between the problem and a distinct body of knowledge, thus revealing a new essential aspect of the problem (Cellucci 2008: p. 206).

Cellucci does not further develop this into a particular aspect of his account, but we do and believe it is important to do so. To emphasize the importance of including epistemic interests, we offer an example from mathematical practice where it makes a difference whether one is rooted in an algebraic or geometrical way or reasoning.

\section{Illustration from Practice}

During the nineteenth century, the majority of mathematicians eschewed the use of diagrams or graphs and had a clear preference for symbolic manipulation in order to prove results. ${ }^{10}$ The downgrading had of course to do with the post-Kantian mistrust

\footnotetext{
${ }^{10}$ For an interesting overview on the status of visualization in historical periods of logic and mathematics, see Mancosu (2005).
} 
of geometric intuition that flowed from failing in facilitating comprehension, which was forcefully illustrated by the conception, e.g. by Weierstrass, of continuous functions that are nowhere differentiable, hence not allowing for any visual representation. Visuals were thus deemed unnecessary at best, misleading at worst. The ideal of formal proof that was developed and embraced from that period was that of a logical sequence of symbolic sentences.

The approach of Weierstrass to mathematics, which insists on logical purity, can be seen as a specific epistemic interest. If we would use Cellucci's notions (of essential aspects to be revealed) in this case, they would need to be aspects about the algebraic nature of mathematical problems:

We shall give a purely arithmetical definition of complex magnitudes. The geometrical representation of the complex magnitudes is regarded by many mathematicians not as an explanation, but only as a sensorial representation, while the arithmetical representation is a real explanation of the complex magnitudes (Weierstrass in a 1874 lecture, quoted from Ferreirós 2007: p. 211. Emphasis added).

It is with these epistemic interests that Weierstrass presented what is now known as the Weierstrass function. Weierstrass believed that algebraic truths give a systematic theoretical foundation for function theory. ${ }^{11}$ This resulted in a proof of continuous non-differentiable functions and continued to abandon visual intuition as a secure guide in the development of mathematics. But not everyone in the mathematical community wished to follow the same path. Von Koch published a paper in 1904 in which he concedes that the example of Weierstrass has shown that continuous and nowhere differentiable functions exist, but he finds an essential aspect to remain hidden:

It seems to me that his example is not satisfactory from the geometrical point of view since the function is defined by an analytic ${ }^{12}$ expression that hides the geometrical nature of the corresponding curve and so from this point of view does not see why the curve has no tangent (Quoted from translation Edgar 1993: p. 26. Emphasis added).

Von Koch's geometrical approach to this problem, which results in his famous Snowflake as a visual representation of an early fractal, was genuinely geometrical. Its construction process revealed the geometrical nature of fractals, which remained hidden in the purely logical proof.

These examples show that there is contextual variation of epistemic interest which would determine when an essential aspect of a mathematical problem is revealed.

\footnotetext{
11 See Weierstrass' letter to his colleague Schwarz, cited from Bottazzini (2001).

12 Not to be confused with Cellucci's use of the term analytic.
} 


\section{Lange}

\section{Account}

Lange (2014) argues that the distinction between explanatory and non-explanatory proofs is in the way the proof extracts a theorem from its premises. The theorem should be seen in terms of a set-up or problem on the one hand and a result on the other hand. For theorems of the kind All F's are G's, the set-up involves F's instantiation and the result involves G's instantiation. Results have a feature that, once salient, prompts for an explanation. Proofs which exploit this feature in the setup are recognized as explaining why the theorem holds.

A first example, based on proofs of a theorem from probability theory, is one where 'a mathematical result that exhibits symmetry of a certain kind is explained by a proof showing how it follows from a similar symmetry in the problem. Each of these symmetries consists of some sort of invariance under a given transformation' (Lange 2014: p. 496. Emphasis added). The core idea is that a proof appealing to an underlying symmetry in the set-up counts as explanatory exactly because the symmetry in the result struck us in the first place. He gives several examples where this feature is a puzzling symmetry, and argues that proofs exploiting a symmetry in the problem are recognized as explaining why the theorem holds. But symmetry is not necessarily the only feature. Other features such as unity and simplicity of the result could likewise be salient. This leads to the question whether, for that feature of the result, a proof can be found which exploits the same kind of feature in the setup.

Lange gives the following example for the feature unity. When one takes an ordinary calculator keyboard, one can see that all numbers belonging to a certain type of 'calculator number ${ }^{13}$, share a certain property. ${ }^{14}$ Lange states that a case-bycase proof treats this as a coincidental result. Obtaining a result that applies to every single calculator number, on the other hand, proceeds from a property common to each of these numbers and shows why the unity of the result is not a coincidence at all.

\section{Epistemic Reading}

Lange's account can, in addition to Steiner's and Cellucci's account, be adapted into our epistemic approach. Firstly, the actual explanation goes beyond the proof. A broader argument consists of:

- Recognizing a salient feature in both the result and the set-up.

- Showing how this feature is invariant to the transformations of the proof.

\footnotetext{
13 A calculator number is here a six-digit number that is constructed by taking the three digits on any row, column or main diagonal of the keyboard in forward and then reverse order. For the detailed overview of this case, see (Lange 2014: pp. 487-489).

14 The property here is being divisible by 37 .
} 
Secondly, this can be restated as abilities concerning understanding why the theorem is the case. Abilities that are central to this account are:

- The ability to recognize a salient feature in both the result and the set-up.

- The ability to demonstrate how a noteworthy feature in the theorem is related to a similar feature in the premises.

\section{Contextual Factors $K$ and $I$}

Lange sees an important contextual aspect in his account. A proof will only be considered as explanatory in a context where an agent is able to recognize a feature as being salient:

The distinction between proofs that explain why some theorem holds and proofs that merely establish that it holds exists only when some feature of the result being proved is salient. That feature's salience makes certain proofs explanatory. A proof is accurately characterized as an explanation (or not) only in a context where some feature of the result being proved is salient (Lange 2014: p. 507).

Undoubtedly, the background and skill corpus affects the ability to recognize such features. As we already mentioned, Lange takes into account that several features can be seen as salient. One of the possible features, next to symmetry, he discusses is unity. In the next section, we give an example of how the recognition of this feature depends on epistemic interests.

\section{Illustration from Practice}

The quest for unity is a strong driving force in mathematical work. Mathematicians strive for theorems that state properties of all the members of classes of interest. A major unifying concept in mathematics, introduced at the end of the nineteenth and beginning of the twentieth century, is that of a set. Ever since, alternative routes have been explored to seek a conceptual framework within which all mathematical results can be viewed. Take, for example group theory and category theory:

Having proved, using only the group axioms, that group inverses are unique, we know that this fact will apply to every single example of a group. No further work is required. If tomorrow you come across a quite new kind of mathematical structure, and you determine that what you have is a group, you will know at once that every element of your group has a single inverse. In fact, you will know that your newly discovered structure possesses every property that can be established - in abstract form - on the basis of the group axioms alone (Devlin 2000: p. 193).

Our theory provides general concepts applicable to all branches of mathematics, and so contributes to the current trend towards uniform treatment of different mathematical disciplines. In particular it provides 
opportunities for the comparison of constructions and of the isomorphism occurring in different branches of mathematics (Eilenberg and MacLane 1945: p. 236).

All of these routes have their own way of tracing unity in mathematical results. Set theory emphasizes membership, while category theory emphasizes function; group theory joins groups by group homomorphisms, while topos theory joins topological spaces by continuous maps. Research in a certain field focuses on a particular type of unity. Moreover, category theory is affectionately known as abstract nonsense due to its highly abstract concepts. This high level of abstraction is shared by other unifying theories, but reference to highly abstract concepts will not always be telling or preferred. It is clear that Lange's example of calculator numbers locates a far more concrete type of unity. Consequently, epistemic interests cannot be detached from the question which unity is considered to be a striking feature and will also designate the direction in which a similar unity in the set-up will be searched.

\section{Embracing Pluralism}

We have seen three accounts of explanation and adapted them to our epistemic and contextual framework. The epistemic account fleshed out types of activities and arguments that form the explanation of the theorem and specified the type of abilities involved to understand the theorem. Illustrations from practice conveyed the importance of including contextual factors such as the epistemic interest.

But this leaves us with an important question. Do we really need all three accounts, and perhaps even other accounts in the philosophical literature, to say something about mathematical explanation? Steiner had the idea that his account could potentially grasp all mathematical explanations: 'Perhaps all mathematical explanations, then, may be treated similarly' (Steiner 1978: p. 148). Lange and Cellucci distance themselves from Steiner and seem to intend, although they do not say so explicitly, to take his place as a potential monist account of mathematical explanation.

At face value, the multiplicity of types of arguments and abilities we explicated in "Accounts of Mathematical Explanation" are all mathematically valuable. Moreover, they are not simply reducible to each other, so there is no single account of explanation that exhausts the arguments or abilities. Some differences are:

- Steiner sees mathematical explanation as being provided within a family of related proofs, and Lange and Cellucci do not require a relationship with other proofs.

- Cellucci's explanatory hypothesis can reveal a connection between entities or structures in the theorem, and Steiner's characterizing property has to be about an entity or structure mentioned in the theorem.

- Lange requires that the striking feature that needs explanation must have a feature of the same kind in the set-up, and Cellucci's explanatory feature can reveal how another feature reveals something essential. 
At this point, we have several accounts of explanation but no clear evidence that one of them captures all cases of explanatory proofs in mathematics. Rather than presupposing that there necessarily is a single overarching account for mathematical explanation, we suggest to follow another path. Instead of restricting ourselves to a monist view, we propose pluralism (about types of explanation and its corresponding understanding) as a heuristic stance. ${ }^{15}$ In principle, every account of mathematical explanation will admit an epistemic, contextual reading with context-dependent claims. Philosophical reflection and the analysis of specific case studies ${ }^{16}$ can be used to further adapt existing accounts of explanation or develop new accounts. It may be the case that in the end of this investigation only one account remains, rendering all others superfluous, but at this point we have no reason to believe that this will be the case. An author who also adopts this kind of position is Pincock:

I will proceed cautiously and set aside the strong claim that there is only one kind of explanatory proof. Instead, I will allow that there might be many ways to arrive at an explanatory proof. My focus, then, is on a restricted class of explanatory proofs that I will call abstract mathematical explanations (Pincock 2015: p. 2).

Hafner and Mancosu (2005) discuss two alternative approaches to investigate the nature of mathematical explanation: top down or bottom up. The former approach consists of starting with a general model of explanation and consequently testing how well they account for mathematical practice. In the latter approach, one avoids any prior commitment to a particular model and tries to find recurrent types of explanation in the practice. They favour the bottom-up approach, because the top down would often mean 'forcing the evidence from mathematical practice into a predefined mould, thereby narrowing the perspective from the outset and probably leading to distortions' (Hafner and Mancosu 2005: p. 221). We are sympathetic to this worry. A top-down monist view would lead to the misunderstanding or disregard of cases of genuine mathematical explanations that do not fit a particular account of explanation. The bottom-up approach, on the other hand, looks at cases from actual mathematical practice without a commitment to a specific account of explanation. It is our view that an optimal use of this approach requires a pluralistic stance: Either this case fits an existing account, which can count as evidence for its value and/or can lead to an adaptation, or this case does not fit an existing account, which gives rise to the proposal of a new account. Hence, the pluralist stance as a heuristic guideline does not entail 'anything goes', where any arbitrary account of explanation is beyond criticism or revision. Rather, it encourages the optimal use of the resources provided by the philosophical literature in order to get a grip on what mathematical explanation could mean.

\footnotetext{
15 See Weber et al (2013) for a similar argument about pluralism towards scientific explanation.

${ }^{16}$ For a discussion about the value of both an analytical and reflective approach to mathematical explanation, see Weber and Frans (2016).
} 


\section{Concluding Remarks}

In the first section, we stipulated that agents can have diverging epistemic interests, but we do not exclude the possibility that they may have diverging understanding interests as well. These interests will determine which type(s) of explanation $\left(\mathrm{E}, \mathrm{E}^{\prime}\right.$, etc) and corresponding understanding ( $\mathrm{U}, \mathrm{U}^{\prime}$, etc) are contextually appropriate for consideration. ${ }^{17}$ Note that we do not want to make the claim that each agent or community must have his own particular account of explanation. Instead, each agent or community may consider one or several types of understanding ( $\mathrm{U}$ as rendered by type of explanation E) as valuable. In short, this would mean that the type or types (plural) of abilities one searches for in understanding (and thus the type of explanation that grants them) will contextually determine the truth of 'Proof $\mathrm{P}$ explains t'. This functions as a third contextual factor.

Once we read explanation as involving a recipient to which it must give understanding (an epistemic, rather than an ontic reading), context-determining factors were brought to the fore. We have presented two contextual factors: (i) corpus-dependent success $\mathrm{K}$ and (iii) epistemic interests I. This entails that the statement 'Theorem $\mathrm{t}$ is explained by proof $\mathrm{P}$ ' is true (or false) in the light of three contextual factors:

(1) Corpus-dependent success Proof P fits with the background/skill corpus K of $\mathrm{S}$ such that it leads to $\mathrm{S}$ possessing abilities (appropriate to understanding why the theorem is true). ${ }^{18}$ This entails that the truth of the above statement varies contextually with type of background/skill corpus of the agent $\left(\mathrm{K}, \mathrm{K}^{\prime}\right.$, etc).

(2) Epistemic interests The abilities granted by proof $\mathrm{P}$ include those that are of epistemic interest. The truth of the above statement varies contextually with the epistemic standards (I, I', etc).

(3) Explanation/understanding interests Proof $\mathrm{P}$ involves type(s) of explanation E which grants understanding $U$, the abilities of which are deemed appropriate to understanding why the theorem is true in this context. Different contexts of mathematical values may determine which type(s) of explanation (E, $E^{\prime}$, etc) and corresponding understanding ( $U, \mathrm{U}^{\prime}$, etc) contextually determine the truth of the above statement.

Because no single account of mathematical explanation has, at present, managed to capture everything that is mathematically valuable about an explanatory proof, we believe it unwise, at present, to limit ourself to any one of them and accept a (heuristic) pluralism about the accounts of mathematical explanation to be considered in dubbing a proof as explanatory.

\footnotetext{
${ }^{17}$ In this paper, we only treated the type of understanding corresponding with a type of explanation, namely understanding why the theorem is true. However, since epistemic interests are also interests about a 'type' of abilities, they could also be called a type of understanding, but the object of understanding is different. If required, one could distinguish between explanatory understanding $(\mathrm{E} / \mathrm{U})$ and understanding what is of epistemic interest $(\mathrm{I} / \mathrm{U})$. We may find other valuable ways to delineate a kind of understanding, but those are outside the scope of our exploration here.

${ }^{18}$ Furthermore, different proofs ( $\mathrm{P}, \mathrm{P}^{\prime}$, etc) may attain the same abilities (U) for different (or even the same) agents.
} 
As mentioned in "Embracing Pluralism", we welcome further analysis of plural accounts of mathematical explanation which can be tested and further developed by case studies. Furthermore, what is at work in these cases deserves more theoretical exploration. Since we consider a background/skill corpus as a relevant factor in determining the context, these considerations are not restricted to a single person, but to all those who share the relevant background/skill corpus. Community, in that sense, seems a more apt word than agent. It also stresses the potentially high intersubjective nature of the contextual factors. The agents of a community thus not only share a relevant background/skill corpus, but an intersubjective agreement about which abilities are valuable. What's more, different communities may overlap with respect to the contextual factors. We believe these considerations to warrant further inquiry.

A possible tool, proposed for facilitating philosophically relevant inquiry in general, is that of 'style', e.g. in Hacking's influential paper (1992). There, Hacking presents the notion of 'style' as an analytic tool for philosophers and historians of science. He takes differing styles to denote particular ways of scientific reasoning. Hacking has made quite some inspiring general observations-e.g. style not necessarily being peculiar to specific man or epoch, or having generalizing versus mere personal meaning - and has tried to distillate from a variety of historical examples any necessary conditions for being a 'style' as specified. We shall here not be going into the general discussion. Suffice it to mention that, as for mathematics, although he does not get into much detail, Hacking points out that it too belongs among, not above or beyond, the sciences, so style is or should as much be an issue there. That is, to be more explicit: Several kinds of style can play a potential role there and not the strictest of mathematical reasonings on the basis of postulation only.

Browsing for literature on this particular topic, however, it appears that references are few and come with big intervals as well as with hardly any connection between them. Only very recently, Mancosu (2010) has undertaken a preliminary attempt at a systematic treatment of the issue, to find, with a touch of irony, that indeed it 'is not one of the canonical areas of investigation in philosophy of mathematics'. More investigation into this notion of style seems fruitful to get a grasp on when communities share a background/skill corpus and epistemic interests and can consequently be interesting for the contextual approach to explanation/ understanding. This can involve, among others, an analysis on the level of persons (e.g. Bishop-style constructivism, Weierstrassian-style calculus), on the level of groups or schools (e.g. the Bourbaki, or unification programs such as the Erlangen or Langlands ones), on the level of nations (e.g. Spengler's 'Western' style or Bieberbach's 'Deutsche Mathematik') or with a more methodological perspective (e.g. direct vs. indirect style or synthetic vs. analytical style).

Acknowledgements The authors thank an anonymous referee for some very helpful comments. The first two authors are indebted to the Fund for Scientific Reseach-Flanders, of which they are PhD fellows (project $1158617 \mathrm{~N}$ and project $11 \mathrm{M} 9214 \mathrm{~N}$ respectively). All authors would also like to acknowledge the support of VUB research project SRP22: Logic and Philosophy of Mathematical Practices. 


\section{References}

Avigad, J. (2008). Understanding proof. In P. Mancosu (Ed.), The philosophy of mathematical practice (pp. 317-353). New York: Oxford University Press.

Bottazzini, U. (2001). From Paris to Berlin: Contrasted images of nineteenth-century mathematics. In U. Bottazzini, \& A. Dahan-Dalmédico (Eds.), Changing images in mathematics: From the French revolution to the new millennium (pp. 31-47). London: Rougledge.

Cellucci, C. (2008). The nature of mathematical explanation. Studies in History and Philosophy of Science Part A, 39(2), 202-210.

Corry, L. (2012). Modern algebra and the rise of mathematical structures. Boston: Birkhäuser.

Craver, C. F. (2014). The ontic account of scientific explanation. In M. I. Kaiser, O. R. Scholz, D. Plenge, et al. (Eds.), Explanation in the special sciences (pp. 27-52). Dordrecht: Springer.

de Regt, H. W., \& Dieks, D. (2005). A contextual approach to scientific understanding. Synthese, 144, $137-170$.

Devlin, K. J. (2000). The math gene: How mathematical thinking evolved and why numbers are like gossip. New York: Basic Books.

Edgar, G. A. (1993). Classics on fractals. Reading, MA: Addison-Wesley Publishing Company.

Eilenberg, S., \& MacLane, S. (1945). General theory of natural equivalences. Transactions of the American Mathematical Society, 58(2), 231-294.

Ferreirós, J. (2007). The rise of pure mathematics as arithmetic with Gauss. In C. Goldstein, N. Schappacher, \& J. Schwermer (Eds.), The shaping of arithmetic after CF Gauss's disquisitiones arithmeticae (pp. 235-268). Dordrecht: Springer.

Hacking, I. (1992). 'Style' for historians and philosophers. Studies in History and Philosophy of Science Part A, 23(1), 1-20.

Hafner, J., \& Mancosu, P. (2005). The varieties of mathematical explanation. In P. Mancosu, K. F. Jørgensen, \& S. A. Pedersen (Eds.), Visualization, explanation and reasoning styles in mathematics (pp. 215-250). Dordrecht: Springer.

Hempel, C. (1965). Aspects of scientific explanation and other essays in the philosophy of science. New York: Free Press.

Khalifa, K. (2016). khaliphilosophy: Ontic, Epistemic, and Pragmatic Models of Explanation. [Blog post] Retrieved from http://khaliphilosophy.blogspot.com/2016/03/ontic-epistemic-and-pragmaticmodels-of.html.

Lange, M. (2014). Aspects of mathematical explanation: Symmetry, unity, and salience. Philosophical Review, 123(4), 485-531.

Mancosu, P. (2005). Visualization in logic and mathematics. In P. Mancosu, K. F. Jørgensen, \& S. A. Pedersen (Eds.), Visualization, explanation and reasoning styles in mathematics (pp. 13-30). Dordrecht: Springer.

Mancosu, P. (2008). Mathematical explanation: Why it matters. In P. Mancosu (Ed.), The philosophy of mathematical practice (pp. 134-149). Oxford: University Press.

Mancosu, P. (2010). Mathematical style. In E. N. Zalta (Ed.), The Stanford Encyclopedia of philosophy (Spring 2010 Edition). Retrieved from http://plato.stanford.edu/archives/spr2010/entries/ mathematical-style/.

Pincock, C. (2015). The unsolvability of the quintic: A case study in abstract mathematical explanation. Philosopher's Imprint, 15(3), 1-19.

Reck, E. H. (2009). Dedekind, structural reasoning, and mathematical understanding. In B. Van Kerkove (Ed.), New perspectives on mathematical practices: Essays in philosophy and history of mathematics (pp. 150-173). Singapore: World Scientific.

Salmon, W. C. (1984). Scientific explanation: Three basic conceptions. In PSA: Proceedings of the biennial meeting of the philosophy of science association (Vol. 1984, No. 2, pp. 293-305). Philosophy of Science Association.

Sierpinska, A. (1994). Understanding in mathematics. London: The Falmer Press.

Steiner, M. (1978). Mathematical explanation. Philosophical Studies, 34(2), 135-151.

Trout, J. D. (2002). Scientific explanation and the sense of understanding. Philosophy of Science, 69(2), 212-233.

Trout, J. D. (2005). Paying the price for a theory of explanation: de Regt's discussion of Trout. Philosophy of Science, 72(1), 198-208.

Van Fraassen, B. C. (1980). The scientific image. Oxford: Oxford University Press. 
Waskan, J. (2011). Intelligibility and the cape: Combatting anti-psychologism about explanation. In Epistemology of modeling \& simulation: Building research bridges between the philosophical and modeling communities. Pittsburgh. Retrieved from http://philsci-archive.pitt.edu/8530/.

Weber, E., \& Frans, J. (2016). Is mathematics for domain for philosophers of explanation? Journal for General Philosophy of Science. doi:10.1007/s10838-016-9332-1.

Weber, E., Van Bouwel, J., \& De Vreese, L. (2013). Scientific explanation. Dordrecht: Springer.

Weber, E., \& Verhoeven, L. (2002). Explanatory proofs in mathematics. Logique et Analyse, 45(179-180), 299-307.

Wilkenfeld, D. A. (2013). Explaining and understanding. Doctoral thesis, The Ohio State University. 